\title{
Effect of Vagal Nerve Blockade on Moderate Obesity with an Obesity-Related Comorbid Condition: the ReCharge Study
}

\author{
John M. Morton ${ }^{1} \cdot$ Sajani N. Shah ${ }^{2}$ • Bruce M. Wolfe ${ }^{3}$ - Caroline M. Apovian ${ }^{4}$. \\ Christopher J. Miller ${ }^{5}$ - Katherine S. Tweden ${ }^{6}$ - Charles J. Billington ${ }^{7}$ • \\ Scott A. Shikora ${ }^{6,8}$
}

Published online: 5 April 2016

(C) The Author(s) 2016. This article is published with open access at Springerlink.com

\begin{abstract}
Background Vagal nerve blockade (vBloc) therapy was shown to be a safe and effective treatment for moderate to severe obesity. This report summarizes the safety and efficacy of vBloc therapy in the prespecified subgroup of patients with moderate obesity.

Methods The ReCharge Trial is a double-blind, randomized controlled clinical trial of participants with body mass index (BMI) of $40-45$ or $35-40 \mathrm{~kg} / \mathrm{m}^{2}$ with at least one obesityrelated comorbid condition. Participants were randomized 2:1 to implantation with either a vBloc or sham device with weight management counseling. Eighty-four subjects had moderate obesity (BMI $35-40 \mathrm{~kg} / \mathrm{m}^{2}$ ) at randomization.

Results Fifty-three participants were randomized to vBloc and 31 to sham. Qualifying obesity-related comorbidities included dyslipidemia (73\%), hypertension (58\%), sleep apnea (33\%), and type 2 diabetes ( $8 \%$ ). The vBloc group achieved a percentage excess weight loss $(\%$ EWL) of $33 \%$ (11\% total weight loss (\%TWL)) compared to $19 \%$ EWL (6\% TWL) with sham at 12 months (treatment difference 14 percentage points, $95 \% \mathrm{CI}$,
\end{abstract}

Katherine S. Tweden

ktweden@enteromedics.com

Stanford School of Medicine, Stanford, CA, USA

Tufts Medical Center, Boston, MA, USA

Oregon Health \& Science University, Portland, OR, USA

Boston University, Boston, MA, USA

3D Communications, LLC, Raleigh, NC, USA

EnteroMedics Inc, St Paul, MN, USA

University of Minnesota, Minneapolis, MN, USA

Brigham and Women's Hospital, Boston, MA, USA
$7-22 ; p<0.0001)$. Common adverse events of vBloc through 12 months were heartburn/dyspepsia and implant site pain; the majority of events were reported as mild or moderate.

Conclusions vBloc therapy resulted in significantly greater weight loss than the sham control among participants with moderate obesity and comorbidities with a well-tolerated safety profile.

Keywords Vagal nerve blocking $\cdot$ Obesity $\cdot$ Moderate obesity $\cdot$ Active implantable medical device $\cdot$ Bariatric surgery $\cdot$ Laparoscopic surgery $\cdot$ Randomized controlled trial

\section{Introduction}

The vagal nerve blockade (vBloc) device was developed to provide an alternative to standard bariatric surgery for patients with BMI of 35 to $45 \mathrm{~kg} / \mathrm{m}^{2}$. Placement of the device is by standard laparoscopic surgical techniques without anatomic alterations of the gastrointestinal tract. Development of vagal blocking was based on prior observations that vagotomy could improve obesity [1], including hypothalamic damage obesity [2], and is thought to help patients control feelings of hunger. Randomized clinical trials have demonstrated clinically meaningful weight loss with vBloc therapy [3-5] and improvement in obesity-related comorbid conditions such as type 2 diabetes mellitus (DM2) [6]. Vagal blockade using the vBloc Maestro System is the first new obesity treatment device to receive FDA approval in 14 years.

Weight loss and safety results from the ReCharge randomized controlled trial, which evaluated vBloc therapy using a rechargeable device, have been reported [4]. Using a mixedeffects analysis of the intent-to-treat population, an estimated mean percentage excess weight loss (\%EWL) of $26 \%(10 \%$ 
TWL) for vBloc and $17 \%$ for sham $(6 \%$ TWL $)(p<0.001)$ at 12 months was demonstrated.

Patients with moderate obesity, BMI $35-40 \mathrm{~kg} / \mathrm{m}^{2}$, particularly those with obesity-related comorbid conditions, is a cohort of interest since health care assessment groups have noted lack of clinical evidence in this population [7]. The ReCharge Trial enrolled an appreciable number of participants with moderate obesity, and this report summarizes safety and efficacy data from the trial through 12 months in this patient group to confirm that the moderate BMI group had similar efficacy and safety results to the overall cohort and address the lack of clinical evidence in this population.

\section{Methods}

\section{Participants}

The ReCharge Trial study design and methods have been described previously [4]. The majority of participants were enrolled at eight sites in the USA, with two additional sites in Australia. Participants were eligible for enrollment if their BMI was $40-45$ or $35-40 \mathrm{~kg} / \mathrm{m}^{2}$ with one or more of the following obesity-related comorbidities: type 2 diabetes mellitus, hypertension, dyslipidemia, sleep apnea syndrome, or obesity-induced cardiomyopathy. This report includes only those participants with BMI $35-40 \mathrm{~kg} / \mathrm{m}^{2}$.

\section{Study Design}

ReCharge is a randomized, double-blinded, sham-controlled trial comparing vBloc to a custom-designed sham device. Randomization occurred at implant in a 2:1 ratio to vBloc and sham in permuted block sizes of 3 or 6 stratified by clinical site and type 2 diabetes mellitus status.

Participants and all follow-up personnel, including the sponsor, were blinded until 12-month visits were completed for all trial participants. The interaction of the surgeon with study participants was limited after randomization until the study was unblinded due to the difference in implantation techniques for the two study arms. The study was not unblinded until all participants finished their 12-month visit and data were verified.

The safety of the study was monitored by an independent data and safety monitoring board, and all serious adverse events (SAEs) were independently adjudicated for relatedness by an independent clinical events committee (CEC). The ethical standards for subject treatment set forth in the Helsinki Declaration of 1975 were followed. Written informed consent was obtained from all study participants. The study received institutional review board (IRB) or ethics committee (EC) approval from Bellberry Limited EC, Scottsdale Clinical Research Institute Scottsdale Healthcare, Tufts Medical Center IRB, Oregon Health \& Science University IRB, Mayo Clinic Rochester
IRB, Stanford University Medical Center IRB, University of Minnesota IRB, Scripps IRB, and Western IRB. The study was registered on clinicaltrials.gov with the identifier NCT01327976.

\section{Intervention}

The Maestro Rechargeable System consists of two implanted components: (1) leads that are placed around the anterior and posterior vagal trunks near the esophagogastric junction using standard laparoscopic surgery and (2) a rechargeable neuroregulator placed subcutaneously on the thoracic wall [8]. The neuroregulator requires in-home transcutaneous charging approximately two times weekly using a transmit coil placed over the neuroregulator.

The sham arm neuroregulator was custom-designed by the sponsor to dissipate electrical charge at a rate identical to the active device. In the sham device, charge was dissipated into resistors within the sham neuroregulator rather than delivering charge to the vagus. No electrodes were implanted and the abdominal cavity was not entered. Skin incisions, placed where trocars would typically be placed, were made to simulate the laparoscopic procedure and support blinding the participant and the follow-up personnel.

The neuroregulator was programmed in both vBloc and sham groups to deliver at least $12 \mathrm{~h}$ of therapy per day. Of note, frequent charging of the devices was required in both groups despite no therapy delivery in the sham group. This was a feature designed to protect study blinding. The protocol prespecified weekly therapy current amplitude level increases over the first month to $6 \mathrm{~mA}$. If needed, the amplitude could be adjusted on an alternate schedule by the follow-up team if the participant experienced any discomfort related to the therapy. All participants were asked to check their battery level daily and to recharge their battery when necessary.

Follow-up visits occurred weekly in the first month, biweekly through month 3, and monthly thereafter. Monthly visits were conducted within a 2 -week window. All patients participated in a weight management program that typically consisted of a 15-min educational discussion at each visit on healthy food choices, exercise, and behavioral modification by a trained dietitian or a research assistant.

\section{Study Objectives}

The primary 12-month efficacy and safety endpoints of the entire study population have been previously reported [4], and overall study outcomes will continue to be assessed for 5 years. The prespecified coprimary efficacy endpoints were as follows: (1) to compare the mean $\% \mathrm{EWL}$ of the vBloc and sham groups (in the entire sample, at a superiority margin of $10 \%$ ) and (2) to assess whether the percentage of participants in the vBloc group with at least $20 \%$ EWL exceeded $55 \%$ 
and whether the percentage with at least $25 \%$ EWL exceeded $45 \%$. The primary safety endpoint was to determine whether the rate of serious adverse events (SAEs) related to the device, implant/revision procedure, or therapy in the vBloc group was less than $15 \%$.

This report focuses on efficacy and safety, through 12 months, in the moderately obese subgroup with BMI of 35 to $40 \mathrm{~kg} / \mathrm{m}^{2}$. We also present the mean comparison using percentage total weight loss (\%TWL) and various commonly cited \% TWL thresholds.

\section{Statistical Analysis}

Mixed-effects regression models were used to conduct analyses on the weight loss data in this moderate BMI cohort using the intention-to-treat (ITT) population [9]. Models were fit with an unstructured covariance matrix and treating time (study visits) as a categorical variable with time-specific contrasts. Data are assumed to be missing at random under these models. Weight loss results are also reported as a complete case analysis.

Weight loss results are not reported using the last observation carried forward (LOCF) method, the primary imputation method for the 12-month results for the entire ITT population [4], due to the limitations in statistical properties of LOCF imputation [9]. All statistical analyses were performed using SAS version 9.3.

\section{Results}

\section{Participant Baseline Characteristics and Disposition}

Of the 162 randomized vBloc participants and 77 sham participants, 53 vBloc and 31 sham participants were moderately obese with comorbidities at baseline. The treatment groups were well balanced on baseline demographics and medical characteristics in the moderately obese subgroup (Table 1). Approximately $80 \%$ of the participants were female, and the mean age was 52 , and the mean BMI was $38 \mathrm{~kg} / \mathrm{m}^{2}$. The most common obesity-related comorbidities were dyslipidemia, hypertension, and obstructive sleep apnea.

The CONSORT diagram for moderately obese participants in the ReCharge Trial is shown in Fig. 1. Four participants in the vBloc group and three in the sham group had withdrawn from the study by the 12-month visit. The four withdrawals in the vBloc group were due to intra-operative exclusions (discovery of prior upper GI surgery, $>5 \mathrm{~cm}$ hiatal hernia, cirrhotic liver, and delayed gastric emptying), so the device was not implanted. The three withdrawals in the sham group occurred after implant and were due to breast cancer in one case and subject decision in two cases. One participant in the vBloc group required surgical revision to correct tilt of the neuroregulator within the subcutaneous pocket; the revision was uncomplicated with the patient
Table 1 Baseline characteristics by treatment group

\begin{tabular}{|c|c|c|}
\hline Characteristic & $\begin{array}{l}\text { vBloc } \\
(n=53)\end{array}$ & $\begin{array}{l}\text { Sham control } \\
(n=31)\end{array}$ \\
\hline \multicolumn{3}{|l|}{ Demographics } \\
\hline Women, no. (\%) & $42(79)$ & $25(81)$ \\
\hline $\begin{array}{l}\text { Age, mean }(\mathrm{SD}) \\
\text { years }\end{array}$ & $53(8)$ & $51(7)$ \\
\hline \multicolumn{3}{|l|}{ Ethnicity, no. (\%) } \\
\hline Hispanic/Latino & $1(2)$ & $2(7)$ \\
\hline \multicolumn{3}{|l|}{ Race, no. (\%) } \\
\hline Caucasian & $46(87)$ & $29(94)$ \\
\hline African-American & $4(8)$ & $1(3)$ \\
\hline Native American & $2(4)$ & $0(0)$ \\
\hline Asian & $1(2)$ & $1(3)$ \\
\hline \multicolumn{3}{|l|}{ General medical } \\
\hline \multicolumn{3}{|l|}{$\begin{array}{l}\text { Body size measures at } \\
\text { implant, mean (SD) }\end{array}$} \\
\hline Height, $\mathrm{cm}$ & $170(10)$ & $170(10)$ \\
\hline Implant weight, $\mathrm{kg}$ & $104(12)$ & $107(11)$ \\
\hline BMI, $\mathrm{kg} / \mathrm{m}^{2}$ & $38(2)$ & $38(1)$ \\
\hline Excess weight $^{\mathrm{a}}, \mathrm{kg}$ & $35(6)$ & $36(5)$ \\
\hline Waist circumference, $\mathrm{cm}$ & $115(11)$ & $117(9)$ \\
\hline $\begin{array}{l}\text { Type } 2 \text { diabetes mellitus, } \\
\text { no. }(\%)\end{array}$ & $4(8)$ & $3(10)$ \\
\hline Hypertension, no. (\%) & $31(59)$ & $18(58)$ \\
\hline Dyslipidemia, no. (\%) & $37(70)$ & $24(77)$ \\
\hline $\begin{array}{l}\text { Obstructive sleep apnea, } \\
\text { no. }(\%)\end{array}$ & $16(30)$ & $12(39)$ \\
\hline
\end{tabular}

${ }^{\text {a }}$ Excess weight was calculated as the difference between the weight at the time of implantation and the ideal body weight corresponding to a BMI of $25 \mathrm{~kg} / \mathrm{m}^{2}$. There were no significant differences in any baseline characteristics between the two treatment groups

released on the same day as the procedure. The 12-month visit completion rates were $87 \%$ in both the vBloc and sham groups.

\section{Weight Loss}

Weight loss expressed as both \%EWL and \%TWL through 12 months is shown in Fig. 2. At 12 months, the estimated mean $\%$ EWL was $33 \%(95 \% \mathrm{CI}, 29-38)$ in the vBloc group and $19 \%(95 \% \mathrm{CI}, 13-24)$ in the sham group in the ITT population, with a corresponding treatment difference between groups of 14 percentage points $(95 \% \mathrm{CI}, 7-22, p<0.0001)$. Estimated mean $\%$ TWL was $11 \%(95 \% \mathrm{CI}, 10-12)$ in the vBloc group and $6 \%$ ( $95 \% \mathrm{CI}, 4-8)$ in the sham group, with a treatment difference of 5 percentage points ( $95 \% \mathrm{CI}, 2-7, p<0.0001)$.

Among the patients completing the 12-month visit (complete case), the mean $\%$ EWL was $34 \%$ in the vBloc group and $20 \%$ in the sham group (treatment difference 14 percentage points; $95 \%$ CI, 4-24, $p=0.009$ ) and mean $\%$ TWL was $11 \%$ in vBloc group and $7 \%$ in the sham group (treatment difference 5 
Fig. 1 CONSORT diagram of the moderately obese subgroup in the ReCharge Trial

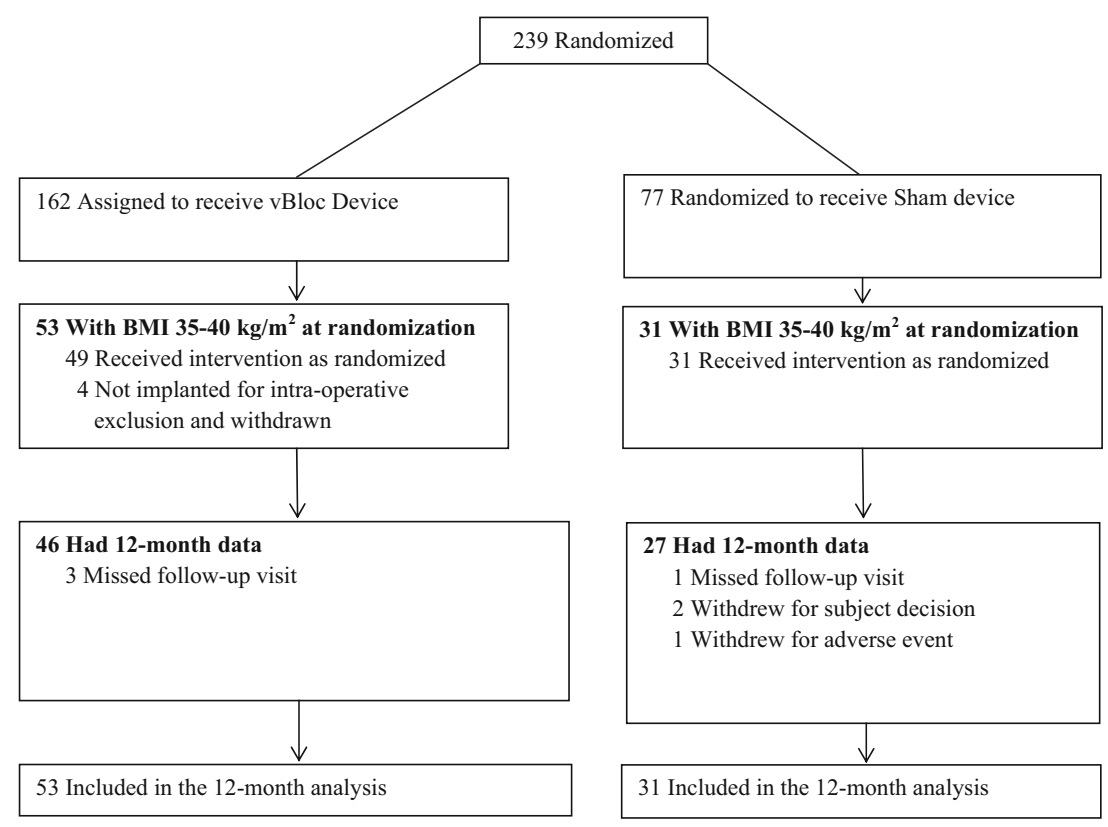

percentage points, $95 \% \mathrm{CI}, 1-8, p=0.010)$. The percentage of patients who achieved categorical weight loss thresholds from $20 \%$ EWL to $50 \%$ EWL and from $7.5 \%$ TWL to $15 \%$ TWL is shown in Table 2. The vBloc group had a higher percentage of patients achieve each level of weight loss, and the relative difference between the treatment groups increased as the threshold of weight loss became more difficult to attain. For example, $50 \%$ of vBloc participants achieved at least $30 \%$ EWL compared to $26 \%$ in the sham group; however, $24 \%$ of vBloc participants achieved the higher threshold of at least $50 \%$ EWL compared to $0 \%$ of participants in the sham group.

\section{Safety}

The adverse event (AE) profile related to device, procedure, or therapy (Table 3) in this subset of the vBloc group was similar to the entire population at 12 months [4]. Heartburn and dyspepsia, neuroregulator site pain, other pain, abdominal pain, incision pain, nausea, eructation/belching, and dysphagia are
Fig. 2 Mean estimated \%EWL and \%TWL through 12 months

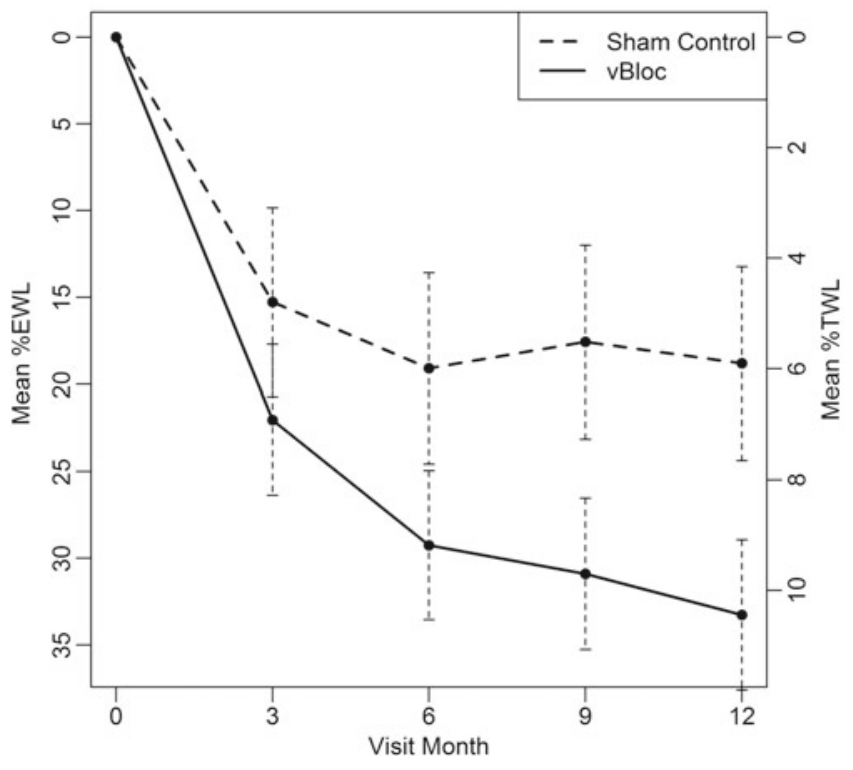

Note: Error bars reflect 95\% confidence intervals. All data are accounted for at each visit using mixed effects regression modeling as noted in the Materials and Methods. At 12 months, 49 of 53 vBloc subjects and 28 of 31 sham participants remained in the trial. 
Table 2 Weight loss responder rates in moderately obese subgroup

\begin{tabular}{llll}
\hline \multirow{2}{*}{ Weight loss achieved } & \multicolumn{2}{l}{ No. $(\%)$} & $p$ value \\
\cline { 2 - 3 } & vBloc $N=46$ & Sham $N=27$ & \\
\hline \% EWL & & & \\
$\geq 20 \%$ & $33(72 \%)$ & $15(56 \%)$ & 0.16 \\
$\geq 25 \%$ & $27(59 \%)$ & $11(41 \%)$ & 0.14 \\
$\geq 30 \%$ & $23(50 \%)$ & $7(26 \%)$ & 0.044 \\
$\geq 40 \%$ & $18(39 \%)$ & $3(11 \%)$ & 0.011 \\
$\geq 50 \%$ & $11(24 \%)$ & $0(0 \%)$ & 0.006 \\
$\%$ TWL & & & \\
$\geq 7.5 \%$ & $31(67 \%)$ & $14(52 \%)$ & 0.187 \\
$\geq 10 \%$ & $22(48 \%)$ & $7(26 \%)$ & 0.065 \\
$\geq 15 \%$ & $13(28 \%)$ & $2(7 \%)$ & 0.033 \\
\hline
\end{tabular}

Responder rates are presented without imputation

the most frequently reported related AEs. More pain at the neuroregulator site was reported in the sham than vBloc group in this subpopulation, though considering the size of the active vBloc and sham devices were identical, this is likely a chance finding due to small sample size. All nonserious AEs in this cohort were reported as mild or moderate in severity, and $84 \%$ of events had resolved at 12 months. Most related events were temporary side effects of the procedure or therapy which resolved with no intervention or with a change in therapy algorithm.

No primary safety endpoint-related SAEs occurred in the moderately obese vBloc participants through 12 months.
Three SAEs $(5.7 \%)$ were adjudicated as related to general intra-abdominal surgery in the vBloc arm. Two events were mild or moderate events of nausea due to anesthesia and one event was discovery of a cirrhotic liver at implant, so the participant was not implanted.

\section{Discussion}

In the subset of participants with a BMI from 35 to $40 \mathrm{~kg} / \mathrm{m}^{2}$ and at least one obesity-related comorbid condition in the ReCharge Trial, weight loss with vBloc therapy was shown to be superior to a rigorous sham control. The vBloc group demonstrated an average weight loss of $33 \%$ EWL or $11 \%$ TWL, with half of participants achieving at least $30 \% \mathrm{EWL}$ and one in four patients achieving at least $50 \%$ EWL. In addition, vBloc therapy had a low rate of associated risks. No primary safety endpoint-related events occurred, and all nonserious AEs were mild to moderate in severity. Most AEs spontaneously resolved or were addressed with modification of the therapy algorithm.

With the combination of clinically meaningful weight loss and favorable safety profile in the moderately obese population, vBloc is a reasonable next step after diet and exercise have failed, or for patients not willing to undergo, or not appropriate candidates for, more aggressive surgical interventions [10-12]. There is currently a treatment gap between those patients who can be successful with diet and exercise and those willing to undergo surgery, so additional efficacious

Table 3 Adverse events related to device, procedure, or therapy through 12 months

\begin{tabular}{|c|c|c|c|c|c|c|}
\hline \multirow[t]{2}{*}{ Adverse event } & \multicolumn{3}{|l|}{ vBloc $N=53$} & \multicolumn{3}{|l|}{ Sham $N=31$} \\
\hline & No. (\%) of patients & No. of events & $\begin{array}{l}\% \text { events } \\
\text { Mild/moderate } \\
\text { Severity }\end{array}$ & No. (\%) of patients & No. of events & $\begin{array}{l}\% \text { events } \\
\text { Mild/moderate } \\
\text { Severity }\end{array}$ \\
\hline Pain, neuroregulator site & $15(28)$ & 17 & 100 & $17(55)$ & 17 & 100 \\
\hline Other & $12(23)$ & 16 & 100 & $3(10)$ & 3 & 100 \\
\hline Heartburn/dyspepsia & $13(25)$ & 13 & 100 & $1(3)$ & 1 & 100 \\
\hline Pain, other & $7(13)$ & 9 & 100 & $0(0)$ & 0 & \\
\hline Pain, abdominal & $5(9)$ & 7 & 100 & $0(0)$ & 0 & \\
\hline Incision pain & $5(9)$ & 6 & 100 & $4(13)$ & 4 & 100 \\
\hline Nausea & $3(6)$ & 6 & 100 & $0(0)$ & 0 & \\
\hline Eructation/belching & $5(9)$ & 5 & 100 & $0(0)$ & 0 & \\
\hline Dysphagia & $4(8)$ & 4 & 100 & $0(0)$ & 0 & \\
\hline Chest pain & $3(6)$ & 3 & 100 & $0(0)$ & 0 & \\
\hline Constipation & $3(6)$ & 3 & 100 & $3(10)$ & 3 & 100 \\
\hline Cramps, abdominal & $3(6)$ & 3 & 100 & $0(0)$ & 0 & \\
\hline Dizziness & $3(6)$ & 3 & 100 & $0(0)$ & 0 & \\
\hline
\end{tabular}

Only adverse events attributed by the investigator to the device, procedure, or therapy that occurred in at least $5 \%$ of vBloc group participants are displayed 
and safe treatment options for obesity and comorbidities are needed. While longer-term efficacy data need to be further studied, the 1-year weight loss reported provides support that vagal blockade may be considered an effective alternative to conventional weight loss surgery particularly in this moderate obesity patient group.

Weight loss in the sham group was greater than expected, which has been discussed elsewhere [4]. Briefly, it was hypothesized that the sham response was due to the robust placebo effect of sham surgery along with daily monitoring and recharging of the sham device. In keeping with our dietary intervention studies, the sham effect was not found to persist in the entire cohort of the ReCharge Trial through 18 months as the sham group regained $40 \%$ of the weight lost at 12 months [5].

The analysis in this report has limitations. First, this is an analysis of a subgroup of the entire randomized sample and has limitations inherent to subpopulations. On the other hand, this is offset somewhat by the fact that this subgroup analysis was prespecified, the analysis was conducted using the ITT population, and there were little missing data. Second, analysis of a subgroup diminishes the statistical power to identify significant differences between the treatment groups; however, statistical superiority in mean weight loss was achieved and most weight loss thresholds were met despite this reduced power. Third, results for this subgroup are presented at 12 months, so the durability of weight loss in this subset of participants requires additional follow-up. Results of the entire ReCharge cohort through 18 months have been published [5] and show that the weight loss among vBloc participants were largely sustained, whereas the sham group regained nearly half of the weight they had lost through 12 months despite remaining blinded through most of the period between 12 and 18 months.

\section{Conclusions}

Individuals with moderate obesity have been considered well-suited candidates for vBloc therapy given that the minimally invasive procedure does not require permanent changes to gastrointestinal anatomy, is reversible, and appears to have a more favorable safety profile to traditional weight loss surgery. In this moderately obese population, the ReCharge Trial demonstrated superior weight loss in the vBloc therapy group than the sham group among participants with moderate obesity. Therapy with vBloc is safe and well tolerated. Additional long-term data and continued follow-up of the ReCharge study are needed to further characterize the safety and effectiveness profile of vBloc therapy in subjects with moderate obesity.

Compliance with Ethical Standards All procedures performed in studies involving human participants were in accordance with the ethical standards of the institutional and/or national research committee and with the 1964 Helsinki declaration and its later amendments or comparable ethical standards.

Informed consent was obtained from all individual participants included in the study.

Conflict of Interest EnteroMedics Inc., the funder/sponsor, was involved in the design and conduct of the study, site selection, and database management. The sponsor provided funding to the clinical sites for patient enrollment, core laboratory analyses, clinical events adjudications, and database entry.

Author 1 has no conflict of interest.

Author 2 received sponsor-provided fees for surgeon training outside of the study conduct.

Authors 3 and 4 received sponsor-provided fees for assistance in preparing and presenting these data to an independent FDA advisory committee.

Author 5: sponsor provided fees for data analysis and critical revision of manuscript.

Authors 6 and 8 are sponsor representatives allowed to review and participate in critical revision of manuscript.

Author 7 received sponsor-provided consulting fees for oversight of the trial (National PI).

Open Access This article is distributed under the terms of the Creative Commons Attribution 4.0 International License (http:// creativecommons.org/licenses/by/4.0/), which permits unrestricted use, distribution, and reproduction in any medium, provided you give appropriate credit to the original author(s) and the source, provide a link to the Creative Commons license, and indicate if changes were made.

\section{References}

1. Kral JG, Gortz L, Hermansson G, et al. Gastroplasty for obesity: long-term weight loss improved by vagotomy. World J Surg. 1993;17:75-9.

2. Smith DK, Sarfeh J, Howard L. Truncal vagotomy in hypothalamic obesity. Lancet. 1983;1:1330-1.

3. Sarr MG, Billington CJ, Brancatisano R, et al. The EMPOWER study: randomized, prospective, double-blind, multicenter trial of vagal blockade to induce weight loss in morbid obesity. Obes Surg. 2012;22:1771-82.

4. Ikramuddin S, Blackstone RP, Brancatisano A, et al. Effect of reversible intermittent intra-abdominal vagal nerve blockade on morbid obesity: the ReCharge randomized clinical trial. JAMA. 2014;312:915-22.

5. Shikora SA, Wolfe BM, Apovian CM, et al. Sustained weight loss with vagal nerve blockade but not with sham: 18-month results of the ReCharge Trial. J Obes. 2015. doi:10.1155/2015/365604.

6. Shikora S, Toouli J, Herrera MF, et al. Vagal blocking improves glycemic control and elevated blood pressure in obese subjects with type 2 diabetes mellitus. J Obes. 2013. doi:10.1155/2013/245683.

7. California Technology Assessment Forum. Controversies in Obesity Management [Internet]. 2015 [cited 2015 Sep 28]. pp. 1- 
140. Available from: http://ctaf.org/reports/controversies-obesitymanagement.

8. Camilleri M, Toouli J, Herrera MF, et al. Intra-abdominal vagal blocking (VBLOC therapy): clinical results with a new implantable medical device. Surgery. 2008;143:723-31.

9. Hedeker D, Gibbons RD. Longitudinal data analysis. Hoboken: Wiley; 2006.

10. Picot J, Jones J, Colquitt JL, et al. The clinical effectiveness and cost-effectiveness of bariatric (weight loss) surgery for obesity: a systematic review and economic evaluation. Health Technol Assess. 2009;13:1-357.

11. Ponce J, Nguyen NT, Hutter M, et al. American society for metabolic and bariatric surgery estimation of bariatric surgery procedures in the United States, 2011-2014. Surg Obes Relat Dis. 2015. doi:10.1016/j.soard.2015.08.496.

12. Sarwer DB, Ritter S, Wadden TA, et al. Attitudes about the safety and efficacy of bariatric surgery among patients with type 2 diabetes and a body mass index of 30-40 kg/m2. Surg Obes Rel Dis. 2013;9:630-5. 